BASIC RESEARCH

\title{
Remote ischaemic postconditioning protects the heart during acute myocardial infarction in pigs
}

\author{
Gyorgy Andreka, Marton Vertesaljai, Gergely Szantho, Gusztav Font, Zsolt Piroth, Geza Fontos, \\ Eszter D Juhasz, Laszlo Szekely, Zsolt Szelid, Mark S Turner, Houman Ashrafian, Michael \\ P Frenneaux, Peter Andreka
}

Heart 2007;93:749-752. doi: 10.1136/hrt.2006.114504

See end of article for authors' affiliations

Correspondence to: Professor Dr M P Frenneaux, Department of Cardiovascular Medicine, The Medical School, University of Birmingham, Edgbaston, Birmingham B 15 2TT,UK; m.p.frenneaux@ bham.ac.uk

Accepted 13 March 2007 Published Online First 19 April 2007
Background: Ischaemic preconditioning results in a reduction in ischaemic-reperfusion injury to the heart. This beneficial effect is seen both with direct local preconditioning of the myocardium and with remote preconditioning of easily accessible distant non-vital limb tissue. Ischaemic postconditioning with a comparable sequence of brief periods of local ischaemia, when applied immediately after the ischaemic insult, confers benefits similar to preconditioning.

Objective: To test the hypothesis that limb ischaemia induces remote postconditioning and hence reduces experimental myocardial infarct size in a validated swine model of acute myocardial infarction.

Methods: Acute myocardial infarction was induced in 24 pigs with 90 min balloon inflations of the left anterior descending coronary artery. Remote ischaemic postconditioning was induced in 12 of the pigs by four 5 min cycles of blood pressure cuff inflation applied to the lower limb immediately after the balloon deflation. Infarct size was assessed by measuring $72 \mathrm{~h}$ creatinine kinase release, MRI scan and immunohistochemical analysis.

Results: Area under the curve of creatinine kinase release was significantly reduced in the postconditioning group compared with the control group with a $26 \%$ reduction in the infarct size $(p<0.05)$. This was confirmed by MRI scanning and immunohistochemical analysis that revealed a $22 \%(p<0.05)$ and a $47.52 \%(p<0.01)$ relative reduction in the infarct size, respectively.

Conclusion: Remote ischaemic postconditioning is a simple technique to reduce infarct size without the hazards and logistics of multiple coronary artery balloon inflations. This type of conditioning promises clear clinical potential.
$\Lambda$ lthough primary angioplasty has reduced the risk of mortality as compared with fibrinolysis in ST elevation myocardial infarction (STEMI; 7\% vs 9\%), left ventricular (LV) dysfunction still occurs in many patients. ${ }^{1}$ The degree of LV dysfunction, the strongest determinant of mortality after STEMI, ${ }^{2}$ has been related to the duration of ischaemia, the number of diseased vessels, the completeness of epicardial coronary artery patency and the restoration of microcirculatory flow, which fails in up to $30 \%$ of patients (no reflow). ${ }^{3}$ However, there is broad consensus that reperfusion in itself also mediates myocardial reperfusion injury. ${ }^{4}$

Warning hearts of impending ischaemia through the delivery of brief tolerating cycles of local ischaemia or ischaemia in remote non-vital organs can reduce infarct size between $40 \%$ and $90 \%$ in various species. ${ }^{5}$ However, the widespread applicability of this preconditioning is limited by the implicit proviso that preconditioning must be instituted before unanticipated ischaemic events. ${ }^{6}$ More recently, the ability to mitigate reperfusion injury through the application of several brief cycles of infarct-associated coronary artery occlusion after a sustained ischaemic insult has been achieved by postconditioning. ${ }^{7}$ Although this controlled reperfusion is successful in animal and even in human studies, ${ }^{8}$ significant methodological hurdles may limit the broad clinical applicability and potential safety of postconditioning. ${ }^{9}$ The objectives of the present study were to investigate whether episodes of ischaemia applied to non-vital remote tissues, immediately after reperfusion in a validated swine model of STEMI, can safely limit infarct size through remote postconditioning.

\section{METHODS}

Experimental animals

All experiments performed in this report conformed to the standards in the Guiding Principles in the Use and Care of Laboratory Animals published by the National Institute of Health (NIH Publication No 85-23, revised in 1996). Twenty four Yorkshire pigs of both sexes, weighing $18-22 \mathrm{~kg}$ and free of clinically evident disease, were randomised to remote postconditioning $(n=12)$ or not $(n=12)$.

\section{Interventional cardiology procedure}

Pigs were anesthetised by ketamine $(0.2 \mathrm{ml} / \mathrm{kg})$, intubated orotracheally, and ventilated mechanically with $\mathrm{O}_{2}$ and $1.5 \%$ isofluorane. The animals were placed on a homeothermic blanket control unit to maintain a core body temperature of at least $37^{\circ} \mathrm{C}$. A $6 \mathrm{~F}$ sheath was inserted into the right femoral artery, heparin was given as an anticoagulant and the left coronary artery was intubated with a 6F JR3.5 guiding catheter. A standard guide wire was introduced into the left anterior descending artery (LAD). Acute myocardial infarction was induced with percutaneous transluminal coronary angioplasty (PTCA) balloon inflation $(2.5 \times 10 \mathrm{~mm}$ Maverick balloon at $6 \mathrm{~atm}$ ) for $90 \mathrm{~min}$ in the LAD distal to the second diagonal branch. Complete LAD occlusion was confirmed by contrast injection. Myocardial infarction was confirmed by ST segment

Abbreviations: $L A D$, left anterior descending artery; $L E$, late enhancement; LV, left ventricule; PTCA, percutaneous transluminal coronary angioplasty; STEMI, ST elevation myocardial infarction 
elevation on the ECG, total creatine kinase release, gadoliniumenhanced contrast MRI scan and histological examination.

\section{Postconditioning procedure}

Immediately after PTCA balloon deflation, reversible lower limb ischaemia was induced with repeated inflation of a blood pressure cuff above the systolic blood pressure of the animals for 5 min followed by lower limb reperfusion by cuff deflation for $5 \mathrm{~min}$. Four cycles of ischaemia-reperfusion were performed.

\section{Enzymatic infarct size}

Blood samples were taken at anaesthetic induction, every $4 \mathrm{~h}$ after deflating the PTCA balloon during day $\mathrm{l}$ and every $6 \mathrm{~h}$ on days 2 and 3. To avoid stress on the animals, they were kept under constant sedation with midazolam. Area under the curve of total serum creatine kinase release (Creatine Kinase Kit, Beckman Coulter, Fullerton, California, USA) was calculated in each animal and used as a surrogate marker of enzymatic infarct size.

\section{Infarct size by MRI scan}

After $72 \mathrm{~h}$, cardiac MRI (1.5-T system, Magnetom Sonata, Siemens Medical Solutions, Malvern, Pennsylvania, USA) was performed using ECG gating under the same general anaesthetic regimen as above. All images were acquired during suspension of the ventilation. After intravenous infusion of gadopentate dimeglumine (Magnevist, Berlex Laboratories, Wayne, New Jersey USA; $0.2 \mathrm{mmol} / \mathrm{kg}$ bolus), late enhancement (LE) imaging was performed with a delay time of $15 \mathrm{~min}$. Volumes of the regions revealing LE were calculated using the ARGUS software (Siemens Medical, Erlangen, Germany).

\section{Infarct size by immunohistochemistry}

After 72 h of reperfusion, animals were killed by using sodium pentobarbital $(160 \mathrm{mg} / \mathrm{kg}$ intravenous) and the heart was excised. The LAD, circumflex artery and right coronary artery were catheterised. LAD was reversibly re-occluded in the original position distal to the second diagonal branch. Evans blue $(0.4 \%)$ was infused into the LAD, circumflex artery and right coronary artery to stain the non-ischaemic myocardium in blue, and thereby outline the area at risk (not blue). The occluded LAD was reopened, a catheter was placed distal to the second diagonal branch and a ligation bend was tightened around the tip of the catheter to occlude the LAD again. 2,3,5triphenyltetrazolium chloride $(0.1 \%)$ at $37^{\circ} \mathrm{C}$ was infused into the occluded part of LAD, which stained the viable myocardium in red to measure the extent of necrosis. The atria and right ventricle were removed and the LV was weighed and then immersed in formaldehyde $(10 \%)$ solution for $24 \mathrm{~h}$. The left ventricle was cut into slices of about $10 \mathrm{~mm}$. Individual slices were weighed and digitally photographed in colour. The extent of myocardial necrosis and the area at risk was quantified in each slice by using image analysis software (Adobe Photoshop V.7.0).

\section{Calculations and statistical analysis}

Data are presented as mean (SEM). Area under the curve for total creatine kinase release was calculated with the following equation:

Area $\left.=\mathrm{A}_{1}+\mathrm{A}_{2}+\mathrm{A}_{3}+\ldots+\mathrm{A}_{14}=\left(\left(\left(\mathrm{y}_{0}+\mathrm{y}_{1}\right) \mathrm{x}_{1}\right) / 2\right)\right)+\left(\left(\left(\mathrm{y}_{1}+\mathrm{y}_{2}\right) \mathrm{x}_{2}\right) / 2\right)$

$$
+\left(\left(\left(\mathrm{y}_{2}+\mathrm{y}_{3}\right) \mathrm{x}_{3}\right) / 2\right)+\ldots\left(\left(\left(\mathrm{y}_{13}+\mathrm{y}_{14}\right) \mathrm{x}_{14}\right) / 2\right)
$$

The area at risk was expressed as a percentage of the LV mass, and the infarct size as a percentage of the area at risk. Significance was assumed if $\mathrm{p}<0.05$ (analysis of variance). All analyses were conducted blinded to the randomisation status (postconditioning or control).

\section{RESULTS}

\section{Enzymatic measurement of infarct size}

The area under the curve (fig 1) of total serum creatine kinase release during the first $72 \mathrm{~h}$ of reperfusion was significantly reduced in the postconditioned group than in the control group; this represents $26 \%$ reduction in the infarct size $(p<0.01$; fig 2$)$. The peak total serum creatine kinase release was also significantly lower in the postconditioned group than in the control group (2659 (113) IU/ml vs 3599 (99) IU/ml; p<0.01). Even at $36 \mathrm{~h}$, a significant difference in total serum creatine kinase release persisted between the postconditioned and the control groups.

\section{Measurement of the infarct size by MRI scan}

Infarct size was measured by the volume of gadolinium LE, which was $22 \%$ lower in the postconditioned group (29 (9) ml vs 38 (11) ml, p<0.05).

\section{Measurement of the infarct size by histological examination}

The proportion of area at risk of the LV was similar between the control and the postconditioned groups (41.3\% (3.4\%) vs $44 \%$ $(4.1 \%)$, non-significant). In the control animals, the infarct size represented $48.4 \%(5.2 \%)$ of the area at risk, whereas in the postconditioned animals, the infarct size represented $23 \%$ $(2.4 \%)$ of the area at risk. This represented a significant decrease in the infarct size $(47.5 \%(5 \%), p<0.01$; fig 3$)$.

\section{DISCUSSION}

The present study confirms the hypothesis that brief cycles of hindlimb ischaemia inducing remote postconditioning applied immediately at the time of reperfusion can reduce the myocardial infarct size by $\sim 48 \%$. This finding is compelling as all the multiple modalities used to assess infarct size consistently shown statistically and clinically significant reductions in the infarct size, one of the key determinants of STEMI prognosis. $^{10}$

In some ways, the findings of this and similar studies represent the logical culmination of two decades of research on conditioning. ${ }^{5}$ Although rapid reperfusion is the best strategy to reduce the complications of myocardial ischaemia, reperfusion itself also confers myocardial dysfunction. ${ }^{4}$ Reperfusion injury involves several mechanisms driven putatively by an overload of calcium and free-radical, including aberrations in myocardial energetics and metabolism, endothelial dysfunction, inflammation, hypercontracture and necrosis/apoptosis. ${ }^{7}$ Although preconditioning has been a source of scientific inspiration, its clinical applicability has been limited by the inability to predict

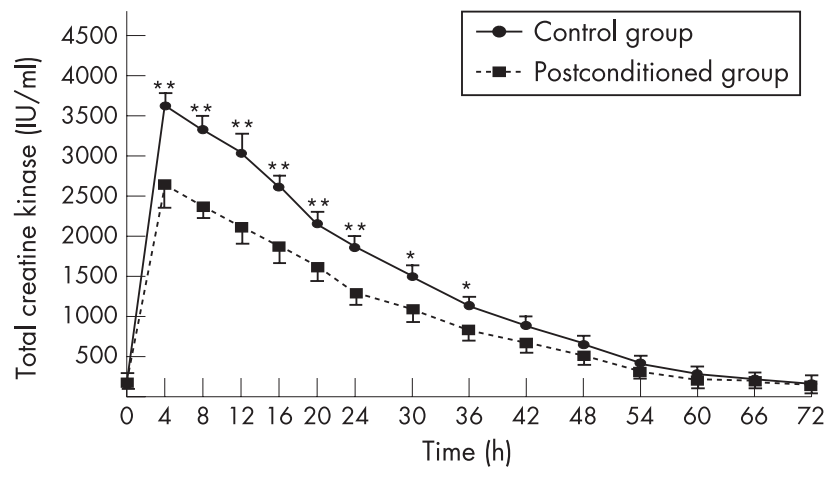

Figure 1 Total serum creatine kinase release. Serum creatine kinase was measured over the first $72 \mathrm{~h}$ of reperfusion (IU/ml) in the control (solid line) and the postconditioned (dotted line) animals. Values are expressed in IU/ $\mathrm{ml}$ as mean (SEM). ${ }^{*} \mathrm{p}<0.05,{ }^{* *} \mathrm{p}<0.01$. 


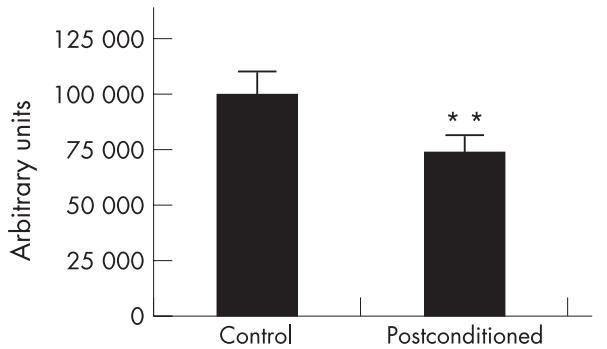

Figure 2 Enzymatic measurement of the reduction in the infarct size. Area under the curve of total serum creatine kinase release was calculated in each animal and used as a surrogate marker of enzymatic infarct size. Values are expressed in arbitrary units as mean (SEM). There was a significant $26 \%$ reduction in the infarct size for postconditioned animals than in control animals $\left({ }^{* *} p<0.01\right)$.

acute myocardial events. The subsequent recognition that activation of reperfusion injury-signalling kinases may reduce the extensive damage of reperfusion and salvage the myocardium $^{11}$ has reinvigorated interest in controlled or mitigated reperfusion-a symptom known in surgical literature for decades. ${ }^{12}$ Although local postconditioning may ultimately prove to be successful, ${ }^{8}$ local coronary balloon inflation may be limited by logistical considerations. ${ }^{9}$ Accordingly, postconditioning has also been recently shown to be effective remotely in rodent models of acute myocardial infarction by potentially invasive renal manipulation ${ }^{13}$ and by limb manipulation in a lapine model. ${ }^{14}$ The latter and present studies show that remote postconditioning (rather than conditioning before reperfusion $)^{13}{ }^{15}$ by ischaemic hindlimb manipulation is safe and effective.

The magnitude of infarct size as determined by the standard measure of myocardial infarction relative to the area at risk as compared with controls was reduced by $\sim 48 \%$. This was confirmed by other validated measures of the infarct size (eg, MRI). ${ }^{16}$ The efficacy of postconditioning in our model is certainly consistent with other studies assessing preconditioning and postconditioning in animals as diverse as rats, rabbits and dogs. ${ }^{17}$ Although preconditioning has both intuitively and empirically been more effective, a direct comparison with a similar pig model of Kharbanda et al ${ }^{18}$ suggests that the reduction in the infarct size is potentially comparable (26 (9)\% vs $53(8) \%, p<0.05$, hence $\sim 50 \%) .{ }^{18}$ Any difference in the absolute infarct size may be explained by methodological differences; Kharbanda et al inflated PTCA balloons in smaller $(\sim 15 \mathrm{~kg})$ Danish Landrace pigs for only $40 \mathrm{~min}$ at the first diagonal. We used larger $(\sim 20 \mathrm{~kg})$ Yorkshire pigs for only 90 min but at the second diagonal, hence the infarct subtended a smaller area at risk. Moreover, we studied our animals at $72 \mathrm{~h}$ rather than immediately.

The most enduring concepts traditionally associated with the conditioning irrespective of the animal model used (rat, rabbit, canine or human) are the duration and number of cycles of ischaemia (single vs multiple cycles) and the organ of index ischaemia (local vs remote; eg, renal ischaemia) is conditioning in general, ${ }^{19}$ and postconditioning in particular, ${ }^{73}$ which must be applied within $1 \mathrm{~min}$ of the reperfusion event. The present study successfully uses a genuine postconditoning regimen in the human-pertinent closed-chest pig model, rather than a small animal model, that has proven to be somewhat resistant to postconditioning, ${ }^{20}{ }^{21}$ and can be inferred that postconditioning is more than just a local hydrodynamic executor, inducing controlled reperfusion. ${ }^{22}$ Instead, remote postconditioning putatively rapidly activates multiple layers of signalling moieties that potently mitigate the ravages of reperfusion injury.
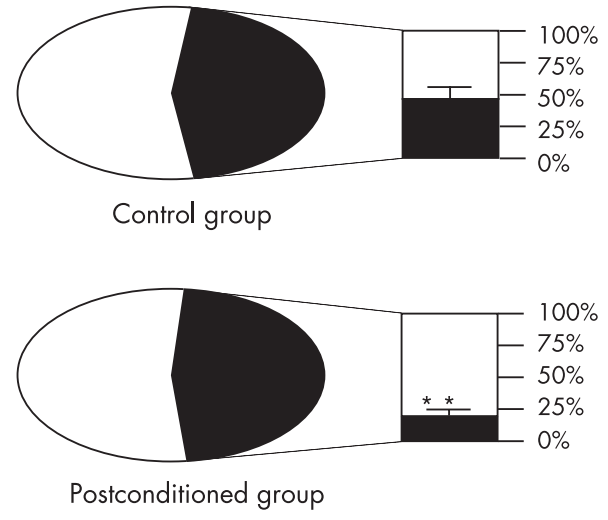

Figure 3 Measurement of the infarct size by histological examination. The $\pi$ graphs show the proportion of area at risk (black)/left ventricle (white) in both the control and the postconditioned animals. The proportion of area at risk did not differ significantly between the control and the postconditioned animals. The bar graphs show the proportion of infarct size (black)/area at risk (white) in both the control and the postconditioned animals. In the control animals, the infarct size represented $48.4 \%(5.2 \%)$ of the area at risk, whereas in the postconditioned animals, the infarct size represented $23 \%(2.4 \%)$ of the area at risk. This represented a significant decrease in infarct size $(47.5(5) \%, p<0.01)$. Values are expressed in percentage as mean (SEM). ${ }^{*} p<0.01$

Although it is likely that the precise mediators and their time course of action will vary (depending on the organ rendered ischaemic and the temporal aspects of the ischaemia) and the mechanisms underlying remote conditioning (preconditioning or postconditioning) remain elusive, it is likely that some similarities exist between the two. It is generally accepted that adenosine release and, hence, activation of the $\mathrm{A}_{2 \mathrm{~A}}$ and $\mathrm{A}_{3}$ receptors have a critical role in the reduction in the infarct size. ${ }^{7}$ As in the case of humoral factors, a neurogenic arc could be one of the triggers easing the release of adenosine in the myocardium in some forms of preconditioning. This reflex can be abolished in some cases by the ganglion blocker, hexamethonium. ${ }^{19}$ Moreover, blocking the subsequent effect of adenosine by non-selective adenosine receptor blocker 8 - $\rho$ sulphophenyl theophylline abolishes the infarct-sparing effect of postconditioning. ${ }^{13}$ Other potential triggers include reactionelaborated reactive oxygen species, endogenous opioids operating through the $\kappa$ and $\delta$ receptors and nitric oxide. Downstream effectors include protein kinase $\mathrm{C}$, reperfusion injury-signalling kinases, $\mathrm{K}_{\mathrm{ATP}}$ channels and the mitochondrial permeability transition pore. It is noteworthy, that unlike humans, pigs do not exhibit an extensive coronary collateral network, hence any putative mediator must be operative through mechanisms other than direct myocardial amelioration, or must be active rapidly after the onset of reperfusion. Clearly, a great deal of mechanistic work remains to be done to elucidate these pathways and how they interact with other conditioning drugs such as inhalational anaesthetics.

Although this observational study is limited by the absence of a definitive mechanistic explanation for genuine remote postconditioning and elucidation of the downstream signalling pathways, the main aim of this study was to establish that remote postconditioning could occur. Indeed, at its inception, postconditioning was considered illogical by some, as the traditional conditioning doctrine required a distinct period of pre-ischaemia and reperfusion for the signalling events to become effective. ${ }^{19}$ Accordingly, this is the first study in a closed-chest large mammal analogous to humans to confirm the ability for this safe and easy form of remote postconditioning to be applied to fields as diverse as paediatric cardiac 
transplantation and primary angioplasty. The present study confirms recent observations in two small animal models of remote postconditioning, an adenosine-mediated model described by Vinten-Johansen's group in rats exposed to a single 5-minute episode of renal ischaemia initiated before reperfusion, ${ }^{13}$ and a lapine model of postconditioning subject to limb ischaemia. ${ }^{14}$ This study highlights the capacity of early remote postconditioning to be of benefit to humans and mandates replication, mechanistic and clinical studies to be performed in order to usher in the decade old paradigm of mechanical conditioning to the bedside.

\section{Authors' affiliations \\ Gyorgy Andreka, Marton Vertesaljai, Gergely Szantho, Gusztav Font, Zsolt Piroth, Geza Fontos, Laszlo Szekely, Zsolt Szelid, Peter Andreka*, Gottsegen Hungarian Institute of Cardiology, Budapest, Hungary \\ Eszter D Juhasz, 2nd Department of Medicine, Semmelweis University, Budapest, Hungary \\ Mark S Turner, Bristol Royal Infirmary, Bristol, UK \\ Houman Ashrafian, Department of Cardiovascular Medicine, University of Oxford, Oxford, UK \\ Michael P Frenneaux*, Department of Cardiovascular Medicine, The University of Birmingham, Birmingham, UK}

*MPF and PA have contributed equally as the principal investigators of this study and are joint senior authors.

Funding: This work was supported by grants from the OTKA (Grant F43380 to P A), the ETT (Grant 302/2003 to P A), the Gottsegen Hungarian Institute of Cardiology (to P A) and the Semmelweis University (to P A). PA is the recipient of the Hungarian Academy of Sciences Bolyai Postdoctoral Fellowship. G Sz is the recipient of the Research Fellowship of the European Society of Cardiology.

Competing interests: None.

\section{REFERENCES}

1 Keeley EC, Boura JA, Grines CL. Primary angioplasty versus intravenous thrombolytic therapy for acute myocardial infarction: a quantitative review of 23 randomised trials. Lancet 2003;361:13-20.

2 Halkin A, Stone G, Dixon S, et al. Impact and determinants of left ventricular function in patients undergoing primary percutaneous coronary intervention in acute myocardial infarction. Am J Cardiol 2005;96:325-31.
3 Ito $\mathrm{H}$, Maruyama A, Iwakura $\mathrm{K}$, et al. Clinical implications of the 'no reflow' phenomenon. A predictor of complications and left ventricular remodeling in reperfused anterior wall myocardial infarction. Circulation 1996;93:223-8.

4 Cannon RO III. Mechanisms, management and future directions for reperfusion injury after acute myocardial infarction. Nat Clin Pract Cardiovasc Med 2005:2:88-94.

5 Yellon DM, Downey JM. Preconditioning the myocardium: from cellular physiology to clinical cardiology. Physiol Rev 2003;83:1113-51.

6 Kloner RA, Rezkalla SH. Preconditioning, postconditioning and their application to clinical cardiology. Cardiovasc Res 2006:70:297-307.

7 Zhao ZQ, Vinten-Johansen J. Postconditioning: Reduction of reperfusion-induced injury. Cardiovasc Res 006, 70:200-11.

8 Staat P, Rioufol G, Christophe P, et al. Postconditioning the human heart. Circulation 2005; 112:2143-8.

9 Laskey WK, Staat $P$, Rioufol G, et al. Letter regarding article by Staat et al, "postconditioning the human heart. Circulation 2006; 113:e665.

10 Sutton MS, Pfeffer AM, Moye L, et al. Cardiovascular death and left ventricular remodeling two years after myocardial infarction : baseline predictors and impact of long-term use of captopril: information from the Survival and Ventricular Enlargement (SAVE) Trial. Circulation 1997;96:3294-9.

11 Yellon DM, Hausenloy DJ. Realizing the clinical potential of ischemic preconditioning and postconditioning. Nat Clin Pract Cardiovasc Med 2005;2:568-75.

12 Okamoto F, Allen BS, Buckberg GD, et al. Reperfusion conditions: importance of ensuring gentle versus sudden reperfusion during relief of coronary occlusion. J Thorac Cardiovasc Surg 1986;92:613-20.

13 Kerendi F, Kin H, Halkos ME, et al. Remote postconditioning. Brief renal ischemia and reperfusion applied before coronary artery reperfusion reduces myocardial infarct size via endogenous activation of adenosine receptors. Basic Res Cardio 2005; 100:404-12

14 Li CM, Zhang XH, Ma XJ, et al. Limb ischemic postconditioning protects myocardium from ischemia-reperfusion injury. Scand Cardiovasc J 2006;40:312-17.

15 Schmidt MR, Smerup M, Konstantinov IE, et al. Intermittent peripheral tissue ischemia during coronary ischemia reduces myocardial infarction: first demonstration of remote ischemic perconditioning. Am J Physiol Heart Circ Physiol. Published Online First 15 Dec 2006. doi: 10, 1152/ajpheart.00617., 2006.

16 Kim RJ, David S, Fieno BS, et al. Relationship of MRI delayed contrast enhancement to irreversible injury, infarct age, and contractile function. Circulation 1999; 100:1992-2002

17 Crisostomo PR, Aairiuko GM, Wang M, et al. Preconditioning versus postconditioning: mechanisms and therapeutic potentials. J Am Coll Surg 2006:202.797-812.

18 Kharbanda RK, Mortensen UM, White PA, et al. Transient limb ischemia induces remote ischemic preconditioning in vivo. Circulation 2002;106:2881-3.

19 Gho BCG, Schoemaker RG, van den Doel MA, et al. Myocardial protection by brief ischemia in noncardiac tissue. Circulation 1996:94:2193-2200.

20 Iliodromitis EK, Georgiadis M, Cohen MV, et al. Protection from postconditioning depends on the number of short ischemic insults in anesthetized pigs. Basic Res Cardiol 2006;101:502-7

21 Schwartz LM, Lagranha CJ. Ischemic postconditioning during reperfusion activates Akt and ERK without protecting against lethal myocardial ischemiareperfusion injury in pigs. Am J Physiol Heart Circ Physiol 2006;290:H1011-18.

22 Heusch G. Postconditioning: old wine in a new bottle? J Am Coll Cardiol 2004;44:1111-12

\section{Save your favourite articles and useful searches}

Use the "My folders" feature to save and organise articles you want to return to quickly-saving space on your hard drive. You can also save searches, which will save you time. You will only need to register once for this service, which can be used for this journal or all BMJ Journals, including the BMJ. 